\title{
Multilevel Models for the Analysis of Angle-Specific Torque Curves with Application to Master Athletes
}

\author{
by \\ Humberto M Carvalho ${ }^{1,2}$
}

The aim of this paper was to outline a multilevel modeling approach to fit individual angle-specific torque curves describing concentric knee extension and flexion isokinetic muscular actions in Master athletes. The potential of the analytical approach to examine between individual differences across the angle-specific torque curves was illustrated including between-individuals variation due to gender differences at a higher level. Torques in concentric muscular actions of knee extension and knee extension at $60^{\circ} \cdot \mathrm{s}^{-1}$ were considered within a range of motion between $5^{\circ}$ and $85^{\circ}$ (only torques "truly" isokinetic). Multilevel time series models with autoregressive covariance structures with standard multilevel models were superior fits compared with standard multilevel models for repeated measures to fit anglespecific torque curves. Third and fourth order polynomial models were the best fits to describe angle-specific torque curves of isokinetic knee flexion and extension concentric actions, respectively. The fixed exponents allow interpretations for initial acceleration, the angle at peak torque and the decrement of torque after peak torque. Also, the multilevel models were flexible to illustrate the influence of gender differences on the shape of torque throughout the range of motion and in the shape of the curves. The presented multilevel regression models may afford a general framework to examine angle-specific moment curves by isokinetic dynamometry, and add to the understanding mechanisms of strength development, particularly the force-length relationship, both related to performance and injury prevention.

Key words: multilevel regression modeling, isokinetic, muscle function, strength.

\section{Introduction}

Physiological functions during exercise, generally used to describe populations, compare groups or define changes across the life span, are all conceptualized in respect to time (Rowland, 2011). In particular, the force-length properties of a whole group of synergetic muscles, i.e., a fundamental issue in muscle mechanics, is generally overlooked despite its practical and theoretical relevance (Herzog et al., 1991). The shape of the curves has been mostly described using maximal voluntary muscular action under isometric conditions (Herzog et al., 1991; Philippou et al., 2004, 2012).

Isokinetic dynamometry has emerged as a favoured method both in clinical research and applied sports contexts to provide information about maximal dynamic muscular action when the velocity of the movement is controlled and maintained constant (Gleeson and Mercer, 1996; Tsepis et al., 2004). Maximal moments of force derived from isokinetic dynamometry data appear to be consistently favored as a measure of isokinetic leg strength. However, the importance of interpreting the profile of isokinetic anglespecific moment curves between the injured (such as anterior cruciate ligament) and healthy lower limb is recognized (Tsepis et al., 2004), but seldom reported and limited to case studies. Also, it is important to consider acute shifts in the forcelength relationship as consequence of muscle-

1 - School of Physical Education, University of Campinas, Campinas, Brazil.

2 - Faculty of Sport Sciences and Physical Education, University of Coimbra, Coimbra, Portugal. 
damaging exercise using angle-specific torque curves and the optimum angle for peak torque generation (Brockett et al., 2001; Brughelli and Cronin, 2007; Philippou et al., 2004; Prasartwuth et al., 2006). Overall, the qualitative and quantitative examination of angle-specific moment curves may be useful in identifying and monitoring patterns of strength development, and potential implications for injury and performance.

Another issue of importance dealing with angle-specific torque curves lies in the nonlinear fitting and the appropriateness of analytical approaches to fit individual and group curves. Both second order (Philippou et al., 2004; Philippou et al., 2009) and fourth order polynomials fitting curves (Prasartwuth et al., 2006) based on repeated measures analysis of variance (ANOVA) and analysis of covariance (ANCOVA) to describe changes in the moments of force across the range of motion have been reported. To the best of our knowledge, no study had examined the impact of different polynomial models fits on the shape of angle-specific torque curves. On the other hand, traditional repeatedmeasures ANOVA to explore longitudinal physiological data may be limited (Kristensen and Hansen, 2004). The restrictive assumptions of repeated-measures ANOVA may not hold when fitting in angle-specific torque curves, in particular the requirement that correlations among measurements on the same subject satisfy a restrictive condition called sphericity, i.e., equal variability of the measurements at each time point and equal correlations between every two measurements on the same individual (Gueorguieva and Krystal, 2004). Multilevel time series models, based on a standard multilevel model for repeated measures data (Goldstein et al., 1994), may be an alternative approach to fit individual angle-specific moment curves. The multilevel regression models allow the use of realistic yet parsimonious variance and consider the correlation patterns in the repeated measures (Goldstein et al., 1994; Singer and Willett, 2003). In particular dealing with multiple measurements that are made close in time may lead to autocorrelation of within-individuals residuals (Goldstein et al., 1994; Steele, 2008). Thus, the application of multilevel time series models, which is an extension of standard multilevel models for repeated measures including an autocorrelation model for within-individual residuals (at Level-1) (Goldstein et al., 1994), may be suitable to fit angle-specific torque curves. In addition, the approach has the flexibility to allow the consideration of group factors and other covariates to study both how the mean values of the curve parameters change across groups and to what extent group differences and the betweenindividual variation can be explained by other factors (Goldstein et al., 1994; Singer and Willett, 2003).

The aim of this paper was to outline a multilevel modeling approach to fit individual angle-specific torque curves describing concentric knee extension (KE) and flexion (KF) isokinetic muscular actions in Master athletes. Furthermore, the analytical method was tested to partition the between-individuals variation accounted to gender differences on the angle-specific torque curves.

\section{Material and Methods}

Sample

The sample included 12 Portuguese Master athletes, 50 to 74 years old engaged in competitive swimming and athletics (female athletes, $n=5,56.0 \pm 3.6$ years; male athletes, $n=7$, $65.5 \pm 5.6$ years). The study was approved by the ethics committee of the Faculty of Sport Sciences and Physical Education, the University of Coimbra, and the subjects were informed about the nature of the study, that participation was voluntary and that they could withdraw from the study at any time.

\section{Isokinetic dynamometry assessment}

In the present study torques in reciprocal concentric muscular actions of KF and KE at $60^{\circ} \cdot \mathrm{s}^{-1}$ from a sample of Master athletes were considered. Isokinetic assessment of reciprocal $\mathrm{KE}$ and $\mathrm{KF}$ actions were made using a calibrated dynamometer (Biodex System 3, Shirley, NY, USA) at angular velocity of $60^{\circ} \cdot \mathrm{s}^{-1}$. The warm-up consisted of 10 min cycling on a Monark cycle ergometer (Monark 814E, Varberg, Sweden) with minimal resistance (basket supported) at 60 rev $\min ^{-1}$ and 2 min of static stretching of the hamstring and quadriceps muscles. For isokinetic assessment, the athlete was placed in a seated position adjusted according to manufacturer guidelines in standardized $85^{\circ}$ hip flexion from the anatomical position. Reciprocal muscular 
actions in the dominant leg were considered. The lever arm of the dynamometer was aligned with the lateral epicondyle of the knee and the force pad was placed approximately 3 to $5 \mathrm{~cm}$ superior to the medial malleolus with the ankle in a plantigrade position. Range of motion during testing was set using voluntary maximal full extension $\left(0^{\circ}\right)$ to $90^{\circ}$ of knee flexion. Cushioning was set using hard deceleration (according to manufacturer guidelines) and therefore, $90^{\circ}$ constituted the range of motion tested. Effects of gravity on the limb and lever arm were accounted for. Athletes gripped handles at the sides of the Biodex System 3 seat during the test procedure. Each athlete performed one set of five continuous maximal repetitions. Visual feedback of moment versus time was provided during the test, but no verbal feedback was given (Baltzopoulos et al., 1991). KE and KF torque values at each angular position from the repetitions with higher maximal torque were retained for analysis and expressed as $\mathrm{N} \cdot \mathrm{m}$. The range of motion considered for analysis was set between 5 and $85^{\circ}$, considering only torques "truly" isokinetic at $60^{\circ} \cdot \mathrm{s}^{-1}$, and torque was averaged in each angular position $(\theta)$. All measurements were made by a single experienced observer (Carvalho et al., 2011).

\section{Statistical analysis}

We initially explored individual trajectories trends fitting ordinary least squares (OLS) estimation to individual data (Figure 1). The basic structure of the repeated measures data in this study had a two-level hierarchical structure with measurements (each $\theta$ within the range of motion) at level 1 nested within individuals at level 2. Thus, the multilevel models described repeated observations over time, in this case over successive $\theta$ (at level 1 ) nested within individuals (level 2).

In the second step of the analysis, we explored the appropriateness of standard multilevel regressions against multilevel time series models. Standard multilevel regressions generally assume that all residuals are normally distributed, and that residuals defined at the same level may be correlated. Multilevel time series models are an extension of standard multilevel models for repeated measures that include an autocorrelation model for within-individual residuals (at level 1) (Goldstein et al., 1994; Steele, 2008).
The third step of the analysis compared the unconditional growth model for the second order polynomial (Equation 1) with higher order polynomial models (Equation 2), up to fourth order polynomials. The unconditional growth model for the second order polynomial is described as:

$$
\begin{gathered}
T_{i j}=t_{0 i}+t_{1 i}\left(\theta_{i j}\right)+t_{2 i}\left(\theta^{2}{ }^{2 i j}\right)+\varepsilon_{i j}, \\
t_{0 i}=\gamma_{00}+\zeta_{0 i} \\
t_{1 i}=\gamma_{10}+\zeta_{1 i} \\
t_{2 i}=\gamma_{20}+\zeta_{2 i}
\end{gathered}
$$

(Equation 1)

Denoted by $T_{i j}$ is the torque at measurement occasion $j(j=1, \ldots, j i)$ for individual $i(i=1, \ldots, n)$, i.e., each $\theta$ (angular position) within the observed range of motion. Denoted by toi is the individual $i$ 's true initial torque value, i.e., the value when the $\theta$ is 0 . Denoted by $t_{1 i}$ is the individual $i$ 's true linear rate of torque change across the observed range of motion. Denoted by $t_{2 i}$ is the individual $i^{\prime} \mathrm{s}$ true nonlinear rate of torque change (second order) across the observed range of motion. $\varepsilon_{i j}$ represents that portion of individual $i$ 's outcome that is unpredicted on the $\theta_{j}$ within the observed range of motion. In the level 2 sub-model, $\gamma_{00}$ represents the population average initial torque value; $\gamma_{10}$ and $\gamma_{20}$ represent the linear and nonlinear (second order) rate of torque change within the observed range of motion. The level 2 residuals are denoted by $\zeta_{0 i}$, $\zeta_{1 i}, \zeta_{2 i}$ that represent deviations of the individual torque change trajectories around their respective group average trend.

The higher order polynomials added to the unconditional growth model are described as:

$$
\begin{aligned}
& T_{i j}=t_{0 i}+t_{1 i}\left(\theta_{i j}\right)+t_{2 i}\left(\theta^{2} i j\right)+t_{3 i}\left(\theta^{3}{ }^{i j}\right)+t_{4 i}\left(\theta^{4}{ }^{4} i j\right)+\varepsilon_{i j}, \\
& t_{0 i}=\gamma_{00}+\zeta_{0 i} \\
& t_{1 i}=\gamma_{10}+\zeta_{1 i} \\
& t_{2 i}=\gamma_{20}+\zeta_{2 i}
\end{aligned}
$$

(Equation 2)

The final step of the analysis explored the inclusion of explanatory variables (covariates). As example in this study we examined gender differences, where male athletes were coded 0 and female athletes were coded 1 , as well as crosslevel interactions (gender $\times \theta$; gender $\times \theta^{2}$ interaction). The explanatory variables were added as fixed effects to equation 2 .

The scale of $\theta$ for KF curves, i.e. movement starting at a position of full leg extension $\left(0^{\circ}\right)$ until flexion of $90^{\circ}$ was set as 
reference in the isokinetic dynamometry assessment. Thus, for interpretation of KE curves, i.e. movement starting at a $90^{\circ}$ position until the full leg extension $\left(0^{\circ}\right)$, the scale of $\theta$ was reversed to allow interpretation of the initial rate of strength with the $t_{1}$ exponent (i.e. represents the linear increase of torque by $\theta$ ) and the $t_{2}$ exponent as an indicator of torque decrease after the attainment of the angle of peak torque.

The reference range of motion was considered in the models for the interpretation of KF curves, i.e., movement starting at a position of full extension $\left(0^{\circ}\right)$ until a flexion of $90^{\circ}$. For the KE, i.e. movement starting at a $90^{\circ}$ position until the full extension $\left(0^{\circ}\right)$, the scale of $\theta$ was reversed to allow interpretation of the initial rate of strength with the $t_{1}$ exponent (i.e. represents the linear increase of torque by $\theta$ ) and the $t_{2}$ exponent as an indicator of the torque decrease after attainment of the angle of peak torque.

Maximum likelihood estimation was used to obtain the parameters. The Akaike's Information Criterion (AIC) and Bayesian Information Criterion (BIC) were reported and used for model comparison. Additionally, visual inspection of residual plotted to determine the models validity to fit torque values throughout the range of motion. Multilevel regression models were obtained using "nlme" package (Pinheiro and Bates, 2000), available as a package in the $R$ statistical language (http://cran.r-project.org).

\section{Results}

The results of model comparison between nonlinear curve fitting with multilevel models to describe KE and KE concentric actions are summarized in Table 1. Smaller value of AIC and BIC for the third degree polynomial model implies that this model was a better fit compared to the second degree polynomial model and fourth degree polynomial model to fit the anglespecific torque curves of isokinetic KE action. As for the isokinetic KF action, the fourth degree polynomial model showed to be the superior fit to describe the angle-specific torque curves. Also, multilevel time series models with autoregressive covariance structures with standard multilevel models had substantially lower AIC and BIC compared with standard multilevel models for repeated measures to fit angle-specific torque curves (multilevel time series model for the best fit $\mathrm{KE}: \mathrm{AIC}=4103.988, \mathrm{BIC}=4162.565$ vs standard multilevel model for comparable KE: AIC = 6348.906, BIC $=6402.601$; multilevel time series model for the best fit KF: AIC $=3426.012$, BIC $=$ 3489.457 vs standard multilevel model for comparable KF: AIC $=5301.213, \mathrm{BIC}=5359.778$ ). The interpretation of estimates and $95 \%$ confidence intervals for initial torque (intercept), the initial rate of torque development (linear $\theta$ exponent), attainment of peak torque (second order exponent) indicate that the second order polynomial estimation underestimated at least the initial rate of torque development and tended to overestimate the angle for maximum torque values attainment (Figure 2 and Table 2).

The inclusion of explanatory variables allows in this example to examine the influence of inter-individual variability of gender on Master athletes' KE and KF angle-specific strength curves. The equations derived from the multilevel regression model with lower AIC and BIC values are presented in Figure 3 and were as follows:

Concentric knee extension $=30.938+6.086(\theta)$ $0.089\left(\theta^{2}\right)+0.0002\left(\theta^{3}\right)-2.466$ (gender $x \theta$ interaction) +0.028 (gender $x \theta^{2}$ interaction)

Concentric knee flexion $=5.978(\theta)-5.771\left(\theta^{2}\right)$ $+0.0002\left(\theta^{3}\right)-0.000011(\theta 4)-1.179$ (gender $x \theta$ interaction) +0.013 (gender $x \theta^{2}$ interaction)

All exponents in the equation were significant at $p<0.01$. The gender term was not included in the equations since $p=0.56$. The intercept term was not included in the equation that described KF, as the term was 2.978 with a standard error of 4.703. The magnitude of the interaction term between gender and the linear term $\theta$ exponent indicates that the male athletes had a higher rate of initial moment of strength, i.e. initial acceleration, as well as a higher peak torque value for both KE and KF. Also, the negative exponent interaction term between gender and the $\theta 2$ suggests a higher decrement of torque after the attainment of peak torque for male Master athletes.

\section{Discussion}

The present study illustrates the use of multilevel time series models to test higher order polynomial fits of individual angle-specific torque curves on $\mathrm{KE}$ and $\mathrm{KE}$ isokinetic muscular actions. 
Table 1

Comparison between nonlinear fits using multilevel modeling with autoregressive covariance structures to describe angle-specific moment curves of concentric knee extension and flexion isokinetic muscular actions in Master athletes

\begin{tabular}{|c|c|c|c|}
\hline Model & $\begin{array}{c}\text { Degrees } \\
\text { of } \\
\text { freedom }\end{array}$ & $\begin{array}{c}\text { Akaike's } \\
\text { Information } \\
\text { Criterion } \\
\end{array}$ & $\begin{array}{c}\text { Bayesian } \\
\text { Information } \\
\text { Criterion } \\
\end{array}$ \\
\hline \multicolumn{4}{|l|}{ Knee extension } \\
\hline $\begin{array}{l}\text { Model 1: } T_{\mathrm{ij}}=\gamma_{00}+\gamma_{10}\left(\theta_{\mathrm{ij}}\right)+\gamma_{20}\left(\theta^{2} \mathrm{ij}\right)+ \\
\zeta_{0 \mathrm{i}}+\zeta_{1 \mathrm{i}}+\zeta_{2 \mathrm{i}}+\varepsilon_{i j}\end{array}$ & 11 & 4130.148 & 4183.843 \\
\hline Model 2: Model $1+t 3 \mathrm{i}\left(\theta^{3} \mathrm{ij}\right)$ & 12 & 4103.988 & 4162.565 \\
\hline Model 3: Model $2+t 4 \mathrm{i}\left(\theta^{4} \mathrm{ij}\right)$ & 13 & 4105.865 & 4169.323 \\
\hline \multicolumn{4}{|l|}{ Knee flexion } \\
\hline $\begin{array}{l}\text { Model 4: } Y_{\mathrm{ij}}=\gamma_{00}+\gamma_{10}\left(\theta_{\mathrm{ij}}\right)+\gamma_{20}\left(\theta^{2} \mathrm{ij}\right)+ \\
\zeta_{0 \mathrm{i}}+\zeta_{1 \mathrm{i}}+\zeta_{2 \mathrm{i}}+\varepsilon_{i j}\end{array}$ & 11 & 3549.111 & 3602.795 \\
\hline Model 5: Model $4+t_{3 \mathrm{i}}\left(\theta^{3}{ }_{\mathrm{ij}}\right)$ & 12 & 3519.852 & 3578.416 \\
\hline Model 6: Model $5+t_{4 \mathrm{i}}\left(\theta^{4}{ }_{\mathrm{ij}}\right)$ & 13 & 3426.012 & 3489.457 \\
\hline
\end{tabular}

$\theta$ represents the angular position

Table 2

Comparison between nonlinear fits using multilevel modeling to describe angle-specific moment curves of concentric knee extension and flexion isokinetic muscular actions in Master athletes

\begin{tabular}{lcc}
\hline & \multicolumn{2}{c}{ Concentric knee extension } \\
\cline { 2 - 3 } $\begin{array}{l}\text { Parameter } \\
\text { (95\% confidence intervals })\end{array}$ & $\begin{array}{c}\text { Second order polynomials } \\
\text { model }\end{array}$ & $\begin{array}{c}\text { Third order polynomials } \\
\text { model }\end{array}$ \\
\hline Intercept & $35.22(19.62$ to 54.86$)$ & $30.91(11.21$ to 50.62$)$ \\
$\theta$ & $4.03(3.78$ to 4.31$)$ & $5.06(4.61$ to 5.51$)$ \\
$\theta^{2}$ & $-0.03(-0.05$ to -0.04$)$ & $-0.08(-0.09$ to -0.07$)$ \\
$\theta^{3}$ & - & $0.0002(0.0001$ to 0.0003$)$ \\
$\theta^{4}$ & - & - \\
\hline
\end{tabular}

\begin{tabular}{lcc}
\hline & \multicolumn{2}{c}{ Concentric knee flexion } \\
\cline { 2 - 3 } & $\begin{array}{c}\text { Second order polynomials } \\
\text { model }\end{array}$ & $\begin{array}{c}\text { Fourth order polynomials } \\
\text { model }\end{array}$ \\
\hline Intercept & $13.63(3.57$ to 23.70$)$ & $8.85(-1.27$ to 19.00$)$ \\
$\theta$ & $2.33(2.13$ to 2.52 & $3.70(3.16$ to 4.27$)$ \\
$\theta^{2}$ & $-0.026(-0.028$ to -0.024$)$ & $-0.12(-0.14$ to -0.09$)$ \\
$\theta^{3}$ & - & $0.002(0.001$ to 0.002$)$ \\
$\theta^{4}$ & - & -0.000011 \\
\hline
\end{tabular}

$\theta$ represents the angular position 
A)

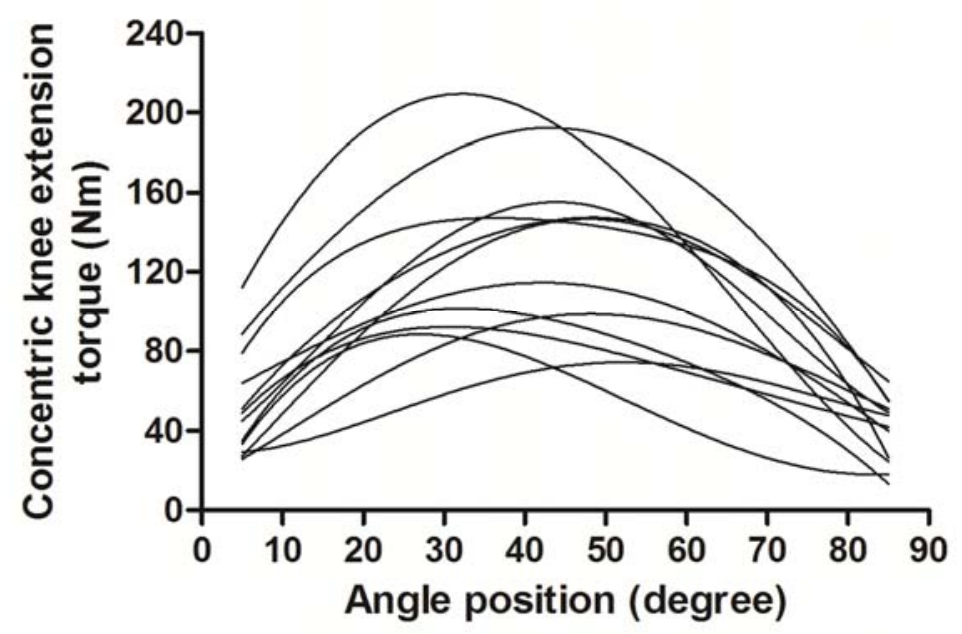

B)

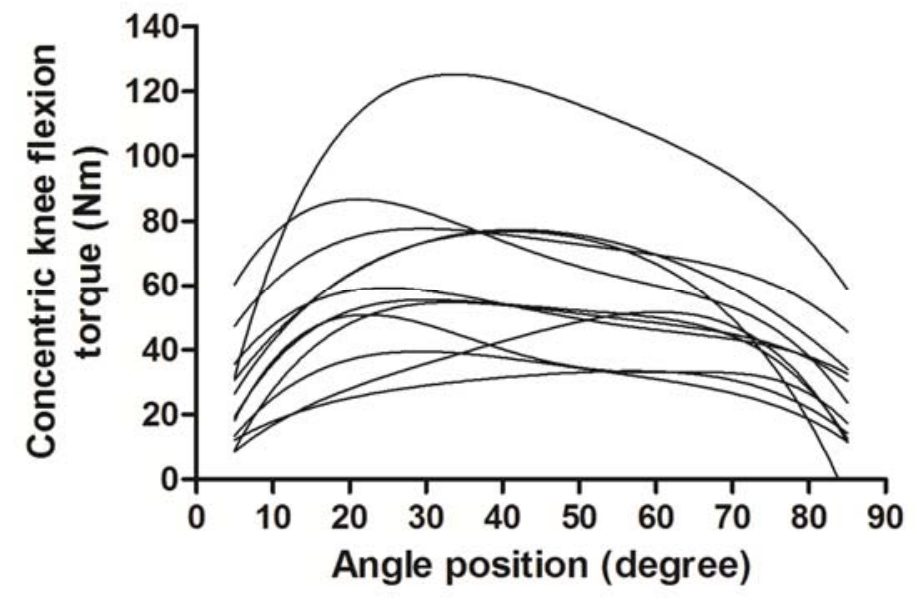

Figure 1

Examining the OLS individual trajectories of isokinetic knee extension (Panel A) and flexion (Panel B) muscular actions at $60^{\circ} \cdot \mathrm{s}^{-1}$ in master athletes.

Note that in Panel A $0^{\circ}$ corresponds to knee flexion at $90^{\circ}$ as the angle scale was inverted in the models for knee extension curves. 
A)

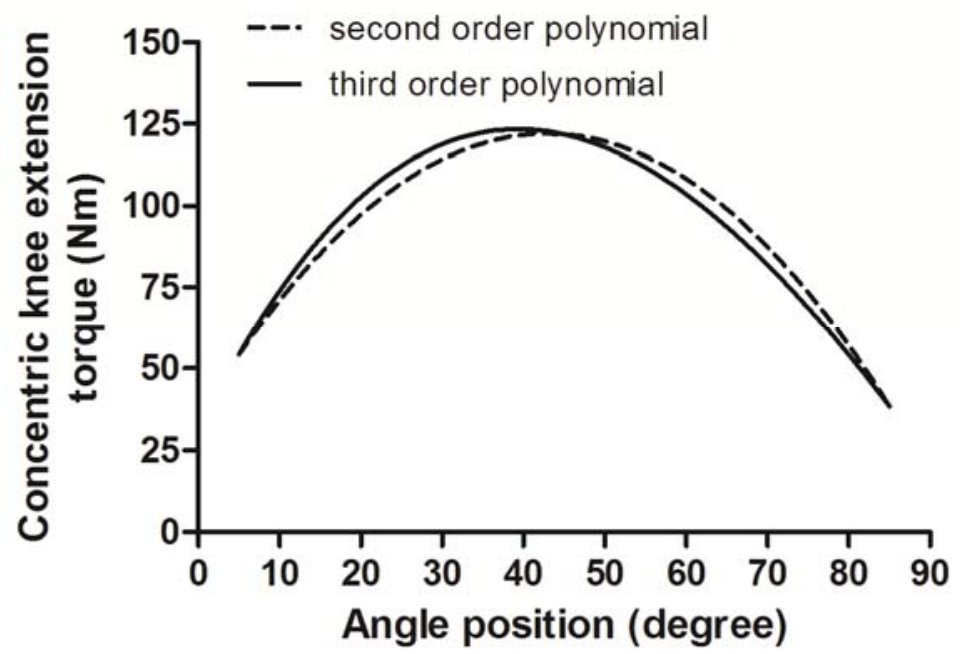

B)

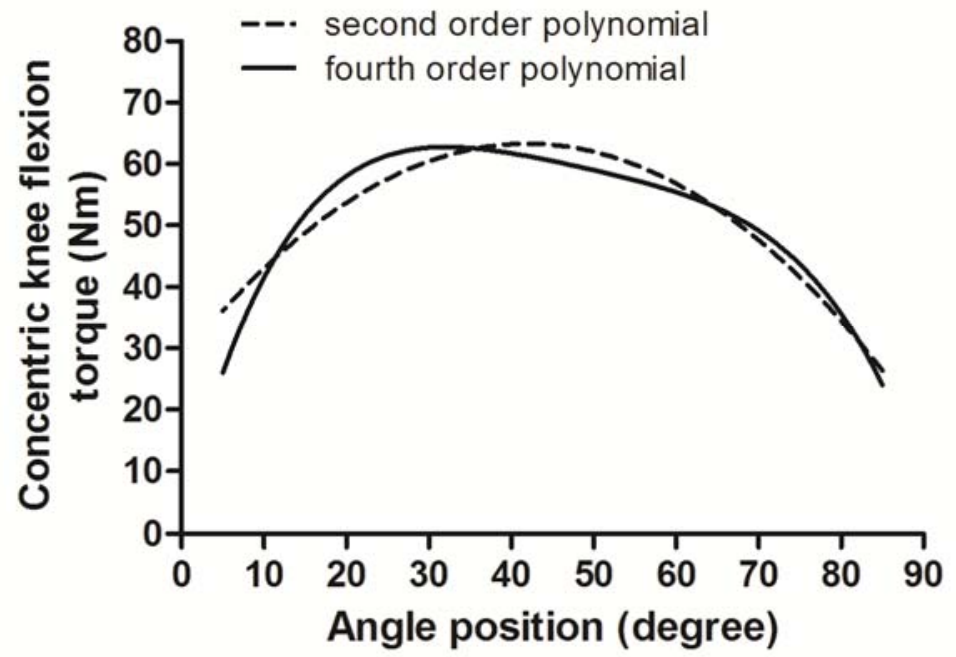

Figure 2

Angle-specific torque curves fitted using multilevel modeling in concentric knee extension and knee flexion isokinetic muscular actions at $60^{\circ} \cdot s^{-1}$ in Master athletes (Panel A: predicted concentric knee extension angle-specific torque curves using second order polynomial and third order polynomial fits, note that $0^{\circ}$ in

Panel A corresponds to knee flexion at $90^{\circ}$ as the angle scale was inverted in the models for knee extension curves; Panel B: predicted concentric knee flexion angle-specific torque curves using second order polynomial and fourth order polynomial fits). 
A)

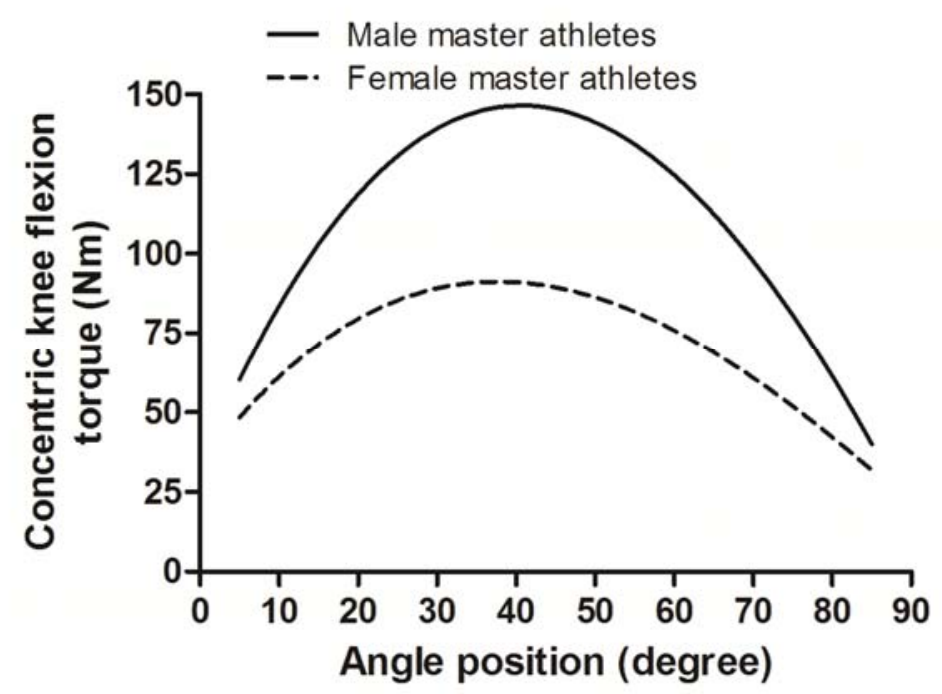

B)

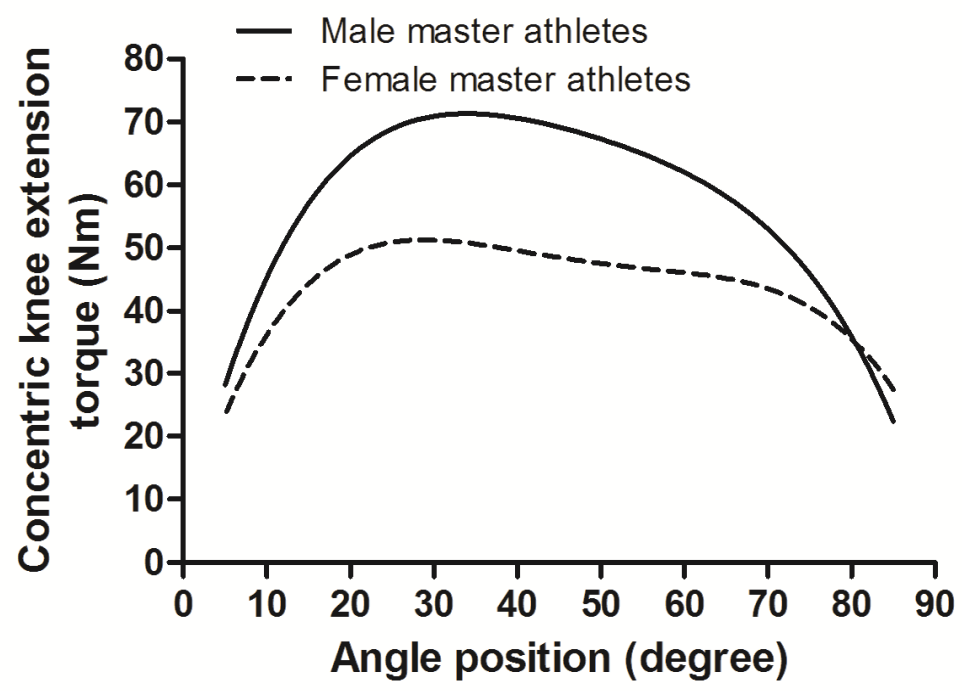

Figure 3

Angle-specific torque curves fitted by gender using multilevel modeling in concentric knee extension and knee flexion isokinetic muscular actions at $60^{\circ} \cdot \mathrm{s}^{-1}$ in Master athletes (Panel A: predicted concentric knee extension anglespecific torque curve, note that $0^{\circ}$ in Panel A corresponds to knee flexion at $90^{\circ}$ as the angle scale was inverted in the models for knee extension curves; Panel B: predicted concentric knee flexion angle-specific torque curve). 
Initial ordinary least-squares fits of individual torque-angle data from the Master athletes are shown in Figure 1 for both isokinetic KE (A) and KF (B). Data for KE ranged from 5.4 to $210.0 \mathrm{~N} \cdot \mathrm{m}$ while flexion data ranged from 126.6 to $0.5 \mathrm{~N} \cdot \mathrm{m}$. Models comparison showed that the third order polynomial fit for KE and the fourth order polynomial fit for KF were superior compared to the second order polynomial model fit. Moreover, the inspection of the $95 \%$ confidence intervals of the intercept, linear and second order exponents suggests that the models produce different estimates for, at least, the initial value, the initial linear rate of torque and the angle of attained peak torque (Figure 2).

The most frequently used curve fitting procedures of angle-specific torque are the Gaussian fitting curve (Bowers et al., 2004; Brockett et al., 2001), the second order polynomial (Philippou et al., 2004; Philippou et al., 2009; Philippou et al., 2012) and the fourth order polynomial (Prasartwuth et al., 2006). The Gaussian fitting curve requires that data is normally distributed. Usually only data points $>75-90 \%$ peak torque can be used to produce the curve (Brughelli and Cronin, 2007). Thus, the method is limited to identify the angle of peak torque. The use of polynomial fitting curve allows describing changes across the entire range of motion (Brughelli and Cronin, 2007). The nonlinear procedures used to fit curves have been based on traditional repeated-measures ANOVA (Philippou et al., 2004; Philippou et al., 2009; Philippou et al., 2012; Prasartwuth et al., 2006; Tsepis et al., 2004). The adoption of the statistical approach may be limited to fit isokinetic anglespecific torque curve due to the restrictive assumptions related to the error covariance matrix. In the present study, the results support the use of third and fourth order polynomials to explore the shape of the angle-specific torque curves. It is apparent that the higher order nonlinear fits may be more sensitive to examine the initial rate of force production, peak torque attained and the angle of peak torque, as well as the rate of decline of torque throughout the range of motion after the angle of peak torque. Particularly, the fit of the third order polynomial for the KE was unexpected, suggesting a trend of shift of the angle of peak to the middle of the range of motion. One of the main advantages of this method is the flexibility to model different error covariance structures, particularly in longitudinal data sets where autocorrelation is often present (Goldstein et al., 1994; Singer and Willett, 2003).

To interpret the fixed exponents of KEcon action, it should be noted that range of motion during testing was set using voluntary maximal full extension $\left(0^{\circ}\right)$ starting at $90^{\circ}$ of knee flexion. Since the angular position in the model was inverted, the linear angular position term refers to the initial acceleration and the second order angular position term refers to attainment of maximum torque production. The magnitude of the second order exponent may also provide an indication of the ability to maintain the torque production throughout the range of motion after peak torque until the full extension (Figure 1).

The analysis of changes in the mechanical properties of the muscle, particularly the shifts force-length relationship in consequence of acute exercise or training exposure has been of interest to researchers (Brughelli and Cronin, 2007; Osternig, 1986). For example, shifts of the optimum angle for peak torque generation in quadriceps muscles towards longer muscle lengths observed after acute eccentric exercise reflect transient changes in the mechanical properties of this muscle (Philippou et al., 2009). Other relevant applied context of interest to researchers and coaches lies in the influence of age-related changes and long-term exposure to the training stimulus in the neuromuscular characteristics of Master athletes, especially in relevance to the increased importance of athletic performance for older athletes (Baker et al., 2010).

The inclusion of explanatory variables allows in this case to examine the influence of inter-individual variability accounted to gender differences on the shape of isokinetic KE and KF concentric actions. Initially, the inclusion of gender in the models showed a substantial variation between athletes in both muscular actions, where it could be inferred that male Master athletes had higher values of torque across the range of motion compared to female athletes. The inspection of cross-level interactions included in the models exemplifies the flexibility of the multilevel time series models to allow interpreting the possible influence of explanatory variables in the different descriptors of the angle- 
specific torque curves. The interactions exponents (gender $x \quad \theta$ interaction and gender $x \quad \theta \quad 2$ interaction) suggest that gender differences have at least an influence on the ability to produce higher rates of initial torque and maximal torques. Differences in maximum torque output, the angle of attainment of maximum torque across the range of motion, particularly for the knee extension, have been reported (Pincivero et al., 2004; Suter and Herzog, 1997; Welsch et al., 1998), although data was mainly obtained by fitting curves to isometric muscles actions. Particularly in Master athletes, the use of the multilevel time series model may allow further inspection of the complexities between age-related changes in muscle architecture, age-related contractile properties changes and training exposure.

The analyses in the present study are confined to describe angle-specific torque with an expected shape. Whether it is better to fit a higher polynomial when the shape of angle-specific torque curves deviates from expected, e.g., after anterior cruciate ligament injury, is a matter that should be further investigated.

In summary, we outlined the use of the multilevel time series model to fit individual angle-specific moment curves. The multilevel regression models provide a flexible approach to deal with structures of short time series, allowing the flexibility to explore individual and/or group characteristics at a higher level as explanatory variables. The procedures described here may provide a general framework to examine anglespecific torque curves by isokinetic dynamomentry both for individual and group characteristics, and add to the understanding of the force-length relationship both related to performance and injury prevention.

\section{Acknowledgements}

The author was supported by a grant from the Coordenação de Aperfeiçoamento de Pessoal de Nível Superior [PNPD/CAPES/2013]. The collaboration of Luis Rama and Carlos Gonçalves is acknowledged by the author.

\section{References}

Baker J, Horton S, Weir P. The masters athlete: understanding the role of exercise in optimizing aging. Routledge, London; New York, p. 204; 2010

Baltzopoulos V, Williams JG, Brodie DA. Sources of error in isokinetic dynamometry: effects of visual feedback on maximum torque. Journal of Orthopaedic and Sports Physical Therapy, 1991; 13: 138-142

Bowers EJ, Morgan DL, Proske U. Damage to the human quadriceps muscle from eccentric exercise and the training effect. Journal of sports sciences, 2004; 22: 1005-1014

Brockett CL, Morgan DL, Proske U. Human hamstring muscles adapt to eccentric exercise by changing optimum length. Medicine and science in sports and exercise, 2001; 33: 783-790

Brughelli M, Cronin J. Altering the length-tension relationship with eccentric exercise : implications for performance and injury. Sports Med, 2007; 37: 807-826

Carvalho HM, Silva MJ, Vaz Ronque ER, Goncalves RS, Philippaerts RM, Malina RM. Assessment of Reliability in Isokinetic Testing Among Adolescent Basketball Players. Medicina (Kaunas), 2011; 47: $446-452$

Gleeson NP, Mercer TH. The utility of isokinetic dynamometry in the assessment of human muscle function. Sports Med, 1996; 21: 18-34

Goldstein H, Healy MJR, Rasbash J. Multilevel time series models with applications to repeated measures data. Statistics in Medicine, 1994; 13: 1643-1655

Gueorguieva R, Krystal JH. Move over ANOVA: progress in analyzing repeated-measures data and its reflection in papers published in the Archives of General Psychiatry. Archives of general psychiatry, 2004; 61: 310-317

Herzog W, Hasler E, Abrahamse SK. A comparison of knee extensor strength curves obtained theoretically and experimentally. Medicine and science in sports and exercise, 1991; 23: 108-114 
Kristensen M, Hansen T. Statistical analyses of repeated measures in physiological research: a tutorial. Advances in physiology education, 2004; 28: 2-14

Osternig LR. Isokinetic dynamometry: implications for muscle testing and rehabilitation. Exercise and sport sciences reviews, 1986; 14: 45-80

Philippou A, Bogdanis GC, Nevill AM, Maridaki M. Changes in the angle-force curve of human elbow flexors following eccentric and isometric exercise. European journal of applied physiology, 2004; 93: 237244

Philippou A, Koutsilieris M, Maridaki M. Changes in kinematic variables at various muscle lengths of human elbow flexors following eccentric exercise. Journal of muscle research and cell motility, 2012; 33: 167-175

Philippou A, Maridaki M, Bogdanis G, Halapas A, Koutsilieris M. Changes in the mechanical properties of human quadriceps muscle after eccentric exercise. In Vivo, 2009; 23: 859-865

Pincivero DM, Salfetnikov Y, Campy RM, Coelho AJ. Angle- and gender-specific quadriceps femoris muscle recruitment and knee extensor torque. Journal of biomechanics, 2004; 37: 1689-1697

Pinheiro JC, Bates DM. Mixed-effects models with Sand and S-plus. Springer New York; 2000

Prasartwuth O, Allen TJ, Butler JE, Gandevia SC, Taylor JL. Length-dependent changes in voluntary activation, maximum voluntary torque and twitch responses after eccentric damage in humans. The Journal of physiology, 2006; 571: 243-252

Rowland T. It's about time. Pediatric exercise science, 2011; 23: 1-2

Singer JD, Willett JB. Applied longitudinal data analysis : modeling change and event occurrence. Oxford University Press, Oxford; New York, 644; 2003

Steele F. Multilevel models for longitudinal data. Journal of the Royal Statistical Society: Series A (Statistics in Society), 2008; 171: 5-19

Suter E, Herzog W. Extent of muscle inhibition as a function of knee angle. Journal of electromyography and kinesiology : official journal of the International Society of Electrophysiological Kinesiology, 1997; 7: 123-130

Tsepis E, Giakas G, Vagenas G, Georgoulis A. Frequency content asymmetry of the isokinetic curve between ACL deficient and healthy knee. Journal of biomechanics, 2004; 37: 857-864

Welsch MA, Williams PA, Pollock ML, Graves JE, Foster DN, Fulton MN. Quantification of full-range-ofmotion unilateral and bilateral knee flexion and extension torque ratios. Archives of physical medicine and rehabilitation, 1998; 79: 971-978

\section{Corresponding author:}

\section{Humberto Moreira Carvalho}

Faculdade de Educação Física, Universidade Estadual de Campinas

Avenida Érico Veríssimo, 701

13083-851 Campinas, São Paulo, BRAZIL,

E-mail: hmoreiracarvalho@gmail.com 